\title{
Neuroinflammation and adult hippocampal neurogenesis in neuropathic pain and alkyl glycerol ethers treatment in aged mice
}

\author{
ANNA TYRTYSHNAIA $^{1,2}$, IGOR MANZHULO ${ }^{1,2}$, YULIA KIPRYUSHINA ${ }^{1}$ and EKATERINA ERMOLENKO ${ }^{1}$ \\ 1،A.V. Zhirmunsky National Scientific Center of Marine Biology', Far Eastern Branch of the Russian Academy of Sciences, \\ 690041 Vladivostok; ${ }^{2}$ School of Biomedicine, Far Eastern Federal University, 690950 Vladivostok, Russia
}

Received November 9, 2018; Accepted March 18, 2019

DOI: $10.3892 /$ ijmm.2019.4142

\begin{abstract}
Neuropathic pain is a condition characterized by unpleasant sensory and emotional experiences associated with a number of diseases or injuries affecting the sensory system through various mechanisms. In this study, we focused on the impact of chronic neuropathic pain on the microglial state and hippocampal neurogenesis in aged mice. In addition, we examined the effects of alkyl glycerol ethers (AGE) treatment on behavioral parameters, hippocampal neuronal and microglial plasticity in aged C57BL/6 mice with neuropathic pain. For the induction of neuropathic pain, we used the model of chronic constriction injury (CCI) of the sciatic nerve. We observed painful behavior in animals subjected to CCI, expressed as a decrease in locomotor activity and the development of cold allodynia. A violation of working and long-term memory was also observed. AGE administration reduced the severity of cold allodynia and prevented memory impairment. In addition to behavioral changes, neuropathic pain was accompanied by microglial activation, changes in the hippocampal production of pro- and anti-inflammatory cytokines, as well as a decrease in neurogenesis. The administration of AGE prevented the neuropathic pain-derived effects, including M1 microglial activation and neurogenesis disruption. However, in vitro experiments demonstrated the pro-inflammatory activation of microglial cells, emphasizing the complexity of the
\end{abstract}

Correspondence to: Dr Anna Tyrtyshnaia, 'A.V. Zhirmunsky National Scientific Center of Marine Biology', Far Eastern Branch of the Russian Academy of Sciences, 17 Palchevskogo Street, 690041 Vladivostok, Russia

E-mail: tyrtyshny.aa@dvfu.ru

Abbreviations: AGE, alkyl glycerol ethers; CCI, chronic constriction injury; DG SGZ, dentate gyrus subgranular zone; DCX, doublecortin; PFA, paraformaldehyde; PCNA, proliferating cell nuclear antigen; ELISA, enzyme-linked immunosorbent assay; ANOVA, one-way analysis of variance

Key words: neuropathic pain, neurogenesis, microglia, cytokines, alkyl glycerol ethers, chimyl alcohol mechanisms underlying the pharmacological effects of AGE. On the whole, the findings of this study demonstrate that AGE treatment prevented behavioral effects of neuropathic pain in mice, and AGE may thus have potential for use in the prevention or treatment of neuropathic pain cognitive and emotional effects. However, as the mechanisms underlying this type of pain are complex, further studies are required to determine the detailed pharmacological effects of AGE.

\section{Introduction}

The International Association for the Study of Pain (IASP) defines neuropathic pain as 'an unpleasant sensory and emotional experience associated with actual or potential tissue damage described in terms of such damage' (1) (https://www.iasp-pain. org/Education/Content.aspx?ItemNumber=1698). Neuropathic pain can be associated with a variety of diseases or injuries affecting the sensory nervous system through various mechanisms. Typically, neuropathic pain is characterized by a wide range of different symptoms and clinical manifestations associated with both sensitivity reduction and enhancement (2).

The mechanisms for the development of neuropathic pain are complex and are not yet fully understood. Sensory information from peripheral structures is processed and transmitted to central nuclei located in several parts of the brain, including the hippocampus (3). The hippocampus is part of the brain limbic system and the main structure that provides spatial and contextual memory, as well as playing an important role in the mechanisms of emotions formation. This area of the brain is associated with the perception of chronic pain, in particular its affective-motivational component (4). Hippocampal neurogenesis in adult animals is known to be involved in learning and memory (5). The dysregulation of adult neurogenesis may result in various neurological disorders, including depression (6), anxiety (7), stress (8) and cognitive disturbance $(9,10)$. Changes in hippocampal plasticity caused by painful stimuli, may result in the development of neuropathic pain, namely cognitive and emotional effects. Pain is a condition characterized by subjective multifaceted symptoms. It has been demonstrated that chronic pain can cause both sensory impairments and various functional disorders (anxiety, amnesia, insomnia and depression) (4). 
The manifestation of pain largely depends on the type of pain, sex (11), the age of the individual (12) and previous experience of pain perception (13). The influence of age on the pain cognitive and emotional consequences can be explained by anatomical and morphological alterations in neurons and glial environments. Such age-related changes are usually associated with an altered neuronal transmission, the sensitivity of receptors involved in the pain perception and modulation. In addition, changes in neuronal electrophysiological parameters, such as the duration of excitatory post-synaptic potentials, synaptic delay and the amplitude of inhibitory postsynaptic potentials, may be crucial $(14,15)$. Alterations in these parameters are primarily associated with the age-related change in the number of excitatory and inhibitory synapses on neurons $(12,16,17)$. The relevance of neuropathic pain studied in aged animals is due to the fact that a large proportion of the population suffering from painful neuropathic disorders refers to an older age group (18).

In our previous study (19), we investigated the effects of neuropathic pain on the microglial state and neurogenesis within the hippocampus in young mice. In this study, we aimed to clarify the mechanisms of behavioral changes characteristic of animals with neuropathic pain. In addition, we examined the effects of alkyl glycerol ethers (AGE) treatment on the parameters under study. This study was devoted to the evaluation of neuropathic pain cognitive effects underlying mechanisms, as well as testing the pharmacologically active substance based on AGE, which can affect the state of hippocampal microglia and neurogenesis.

\section{Materials and methods}

Animals and surgery. Experiments were performed as previously described (19) using 28 18-month old male C57BL/6 mice with an average weight of $45 \mathrm{~g}$. The animals were housed 2-4 per cage with a 12-h light/dark cycle and had ad libitum access to food and water. In order to reduce the amount of stress place on the animals, the mice were handled for only $5 \mathrm{~min}$ once a day over a period of 5 consecutive days prior to the experiments. In addition, glial cultures were obtained from the cerebral cortex of 7 1-day-old Wistar rat pups. The animals were raised at the 'A.V. Zhirmunsky National Scientific Center of Marine Biology', Far Eastern Branch of the Russian Academy of Sciences (Vladivostok, Russia). All procedures performed using animals were approved by the Animal Ethics Committee at the National Scientific Center of Marine Biology Far Eastern Branch, Russian Academy of Sciences (Vladivostok, Russia) according to the Laboratory Animal Welfare guidelines. Neuropathic pain was induced using the model of chronic constriction injury (CCI) of the sciatic nerve, as previously described (20). In brief, the animals were anesthetized with sodium pentobarbital $[50 \mathrm{mg} / \mathrm{kg}$, administered intraperitoneally (i.p.)]. Subsequently, the right sciatic nerve was exposed and 3 ligatures ( 3 silk gut sutures; Ethicon US, LLC., Virginia Beach, VA, USA) were placed around the nerve proximal to the trifurcation at a distance of $1 \mathrm{~mm}$ between each ligature. The ligatures were tightened loosely until a slight twitching of the ipsilateral hindlimb. The animals in the sham-operated group underwent surgery identical to that described above, but without nerve ligation.
Preparation of AGE. The liver from the squid Berryteuthis magister was obtained from Nakhodka Active Marine Fishery Base (Nakhodka, Russia) and stored at $-200^{\circ} \mathrm{C}$. The extraction of total lipids was performed as previously described (19) and as described in the study by Bligh and Dyer (1959); the saponification of lipids was carried out using a conventional technique (21). Following hydrolysis and acidification, the lipid mixture was dissolved in acetone at a ratio of 1:5 at room temperature and incubated for $24 \mathrm{~h}$ at $-20^{\circ} \mathrm{C}$, as previously described (22). The recrystallization of the obtained sediment was performed for the complete separation of AGE from the other lipids. The AGE content in the resulting product was $>99 \%$, where chimyl alcohol was the main component (94\%) (19).

Animal treatment. The AGE preparation was administered to the mice as a water emulsion at a dose of $250 \mathrm{mg} / \mathrm{kg}$ by oral gavage, as previously described (19). The period of administration was 2 weeks from the day that the surgery took place. The mice $(\mathrm{n}=28)$ were divided into 4 different groups as follows: i) The sham-operated group $(n=7)$ in which the mice were treated with the vehicle (water); ii) the CCI group $(n=7)$ in which the animals subjected to sciatic nerve constriction injury were treated with the vehicle (water); iii) the CCI + AGE group $(\mathrm{n}=7)$ in which the mice subjected to sciatic nerve constriction injury were treated with AGE; and iv) the AGE group ( $n=7)$ in which the sham-operated mice were treated with AGE. The animals were sacrificed 14 days after the surgery took place on the day of the final administration of AGE.

Behavioral tests. All the mice were exposed to behavioral tests 2 weeks after surgery the took place, as previously described (19). The determination of thermal hyperalgesia was carried out on a weekly basis. All the tests were performed during the light cycle between 7:00 a.m. and 7:00 p.m. In order to minimize olfactory cues from previous trials, each apparatus was thoroughly cleaned with $10 \%$ ethanol after each use on each animal. In order to allow the animals to adapt and to minimize the stress placed on the animals associated with their new environment, the animals were placed into the test apparatus for $10 \mathrm{~min}$ for 3 days prior to the day of the test. On the day of the test, the mice were left in their home cages in the room used for the experiment for $2 \mathrm{~h}$ prior to the onset of the behavioral test. All behavioral tests were performed on days 13-14 of the experiment. The testing of cold allodynia was carried out on the $6,9,11$ th and 14 day of the experiment.

Thermal allodynia. Thermal allodynia was measured using the hot plate test (Cold/Hot Plate Analgesia Meter no. 05044; Columbus Instruments, Columbus, OH, USA), as previously described $(19,23)$. The test was carried out in a chamber with $30-\mathrm{cm}$ acrylic walls on a metal plate with dimensions of $30 \times 30 \mathrm{~cm}$. The temperature of the cold plate was maintained at $+4^{\circ} \mathrm{C}$, and the cut-off latency was $60 \mathrm{sec}$. The mice were placed on the cold plate, and the total lifting time of the injured hind paw was recorded. The contact time of the limb with the cold plate is significantly reduced in the presence of damage accompanied by pain response (24).

Spontaneous locomotor activity. Locomotor activity was evaluated as previously described (19) by placing each mouse 
into the center of a clear Plexiglas circular open-field arena (diameter, $60 \mathrm{~cm}$; height, $40 \mathrm{~cm}$ ) and allowing the mouse a period of $5 \mathrm{~min}$ to explore its surroundings. Bright overhead lighting was approximately 500 lux inside the arena. The area of the chamber was divided into 37 squares. The behavior of the mice was continuously monitored and recorded using a video camera which was placed over the apparatus. In the subsequent analysis, the number of crossed squares was determined.

Working memory. The working memory of the mice in this study was examined as previously described (19) using the Y-maze spontaneous alternation test. Y-maze testing was carried out using an apparatus with 3 equal arms (length, $30 \mathrm{~cm}$; width, $10 \mathrm{~cm}$; and height, $40 \mathrm{~cm}$ ), made of opaque acrylic glass. Each mouse was placed at the center of the maze and was given a period of 5 min with which to explore its new environment. An arm entry was scored when the mouse entered the arm with all 4 paws. The total number of entries $(\mathrm{N})$ and the number of 'correct' triplets ( $\mathrm{M}$, consecutive choices of each of the 3 arms without re-entries) was evaluated. The alternation rate was computed according to the following formula: $\mathrm{R}(\%)=\mathrm{Mx} 100 /(\mathrm{N}-2)$, as previously described (25).

Novel object recognition. The novel object recognition test was carried out according to the method described in the study by Bevins and Besheer (26). During the training session, each animal was placed for $10 \mathrm{~min}$ into a chamber with 2 identical plastic objects located on the left and right sides of the arena. The animal was then returned to the home cage for a $60-\mathrm{min}$ (for the testing of short-term memory) or 24-h (for the testing of long-term memory) retention interval. For testing, each animal was placed in a test arena, where one of the items was replaced with a new one. The animal was placed equidistantly from both objects. The animal was then left in the testing chamber where it could freely move behind the 2 objects for 5 min. Mouse behavior was continuously recorded using a video camera which was placed over the apparatus. The criterion of the exploration of the object was the location of the nose of the animals at a distance not $>2 \mathrm{~cm}$ from the object. The discrimination ratio was calculated as the time spent on a new object divided by the total time spent exploring both objects. The objects and arenas were carefully cleaned with $70 \%$ ethanol between the tests.

Immunohistochemistry. The collection of material for subsequent histological analysis was performed as previously described (19) on day 14 after the surgery. The mice were anesthetized with sodium pentobarbital (50 $\mathrm{mg} / \mathrm{kg}$, administered i.p.) and then received a transcardial perfusion with $100 \mathrm{ml}$ of ice-cold saline $\left(\sim 4^{\circ} \mathrm{C}\right.$; $(\mathrm{pH} 7.2)$. The mouse brain were immediately removed and divided into 2 hemispheres. The right hemisphere was post-fixed for $12 \mathrm{~h}$ at $4^{\circ} \mathrm{C}$ in fresh-buffered $4 \%$ paraformaldehyde (PFA). The hippocampus from the left hemisphere was extracted and frozen at $-70^{\circ} \mathrm{C}$ for further use in biochemical analysis. The use of this method allows the use of material obtained from the same animal for both immunohistochemical and biochemical analyses. Following fixation with PFA, the tissue samples were embedded in paraffin blocks and sectioned at a thickness of $10 \mu \mathrm{m}$, using a Leica rotary microtome (RM 2245; Leica Biosystems, Buffalo Grove, IL, USA). Following deparaffinization, sagittal paraffin-embedded sections were incubated in $3 \%$ hydrogen peroxide to block endogenous peroxidase prior to immunohistochemical staining. Following 3 washes in $0.1 \mathrm{M}$ phosphate buffer $(\mathrm{pH} 7.2)$, the sections were treated for $60 \mathrm{~min}$ in a $2 \%$ bovine serum albumin solution (sc-2323; Santa Cruz Biotechnology, Santa Cruz, CA, USA) and $0.25 \%$ Triton X-100 (Sigma, St. Louis, MO, USA). The slices were then incubated with primary antibodies (anti-Iba-1 rabbit polyclonal antibodies, 1:500, ab108539; anti-CD86 rabbit monoclonal antibodies, 1:1,000, ab53004; anti-doublecortin antibody, 1:500, ab18723; anti-PCNA mouse monoclonal antibodies, 1:500, ab29; NeuN rabbit monoclonal antibodies 1:1,000, ab177487) (all from Abcam, Cambridge, MA, USA) on a glass slide in a humidified chamber at $4^{\circ} \mathrm{C}$ for $24 \mathrm{~h}$. Following 3 washes with $0.1 \mathrm{M}$ phosphate buffer ( $\mathrm{pH}$ 7.2), the sections were incubated in a secondary antibody (anti-rabbit conjugated to horseradish peroxidase, 1:100, PI-1000; anti-mouse conjugated to horseradish peroxidase, 1:100, PI-2000, Vector Laboratories, Burlingame, CA, USA; fluorescent anti-mouse, 1:500, ab150108; fluorescent anti-rabbit, 1:500, ab150080) (both from Abcam) solution for $45 \mathrm{~min}$. After washing with $0.1 \mathrm{M}$ phosphate buffer ( $\mathrm{pH} 7.2$ ), sections were treated for 5-10 min with chromogen (DAB Plus; Thermo Fisher Scientific, Waltham, MA, USA) to elicit the immunoperoxidase reaction. The slices were subsequently washed with $0.1 \mathrm{M}$ phosphate buffer ( $\mathrm{pH}$ 7.2), dehydrated and embedded in Dako Toluene-Free Mounting Medium (CS705; Dako, Carpinteria, CA, USA).

Immunostaining. In order to evaluate the activity of microglia/macrophages in the mouse hippocampi, immunostaining was performed using anti-Iba-1 rabbit polyclonal antibodies (1:500, ab108539) and anti-CD86 rabbit monoclonal antibodies (1:1,000, ab53004; both from Abcam, Cambridge, MA, USA). For the analysis of neurogenesis, the determination of the number of newly-formed neurons in the dentate gyrus (DG) subgranular zone (SGZ) was performed using anti-doublecortin (anti-DCX) antibody (1:500, ab18723; Abcam). Appropriate secondary antibodies conjugated to horseradish peroxidase (PI-1000, anti-rabbit; PI-2000, anti-mouse) were used according to the manufacturer's instructions (1:100; Vector Laboratories, Burlingame, CA, USA). Proliferating cell nuclear antigen (PCNA)-immunoreactivity was determined using anti-PCNA mouse monoclonal antibodies (1:500, ab29) and NeuN rabbit monoclonal antibodies (1:1,000, ab177487; both from Abcam) in the DG SGZ. Appropriate fluorescent secondary antibodies (anti-mouse, ab150108; anti-rabbit, ab150080) were used according to the manufacturer's instructions (1:500; Abcam). Following incubation with the antibodies $\left(4^{\circ} \mathrm{C}, 24 \mathrm{~h}\right.$ for primary antibodies and room temperature, $45 \mathrm{~min}$ for secondary antibodies), the sections were embedded in Fluoroshield mounting medium with DAPI (ab104139; Abcam).

Image analysis. For immunoperoxidase, staining images were obtained on a Zeiss AxioScope A1 microscope equipped with an AxioCam 503 color and AxioVision software (Carl Zeiss, Oberkochen, Germany). For immunofluorescence staining, the 
slides were examined under a confocal laser scanning microscope (LSM 710 META; Carl Zeiss). Microphotographs were captured and stored as TIFF files. Images were processed and analyzed using ImageJ software (NIH, Bethesda, MD, USA). For DCX and PCNA quantification, every 6th section through the entire brain hemisphere was used. The cells within the SGZ were counted on both blades of the DG. The calculation was performed using only whole cells containing visible nuclei. The DG area was multiplied by the section thickness and expressed in cubic millimeters. The number of immunopositive cells per $\mathrm{mm}^{3}$ was calculated using the following formula: $\mathrm{d}=\left(10^{6} \mathrm{xn}\right) /(\mathrm{Sxl})$, where ' $\mathrm{d}$ ' indicates the cell density, ' $n$ ' the number of immunopositive cells, ' $S$ ' the SGZ area $\left(\mu \mathrm{m}^{2}\right)$, ' 1 ' the thickness of the slice and ' $10^{6}$ ' the coefficient for converting $\mu \mathrm{m}^{2}$ into $\mathrm{mm}^{2}$.

The quantification of Iba1- and CD86-immunopositive cells was performed using every 6th section. To this end, micrographs were processed using the following steps: Converting the image to grayscale mode, background subtraction and binarization. Subsequently, the area occupied by Iba-1- and CD86-immunopositive microglia within the CA1 hippocampal region was calculated. All measurements were performed by an operator who was blinded to the identity of the sections.

Cell culture. Primary microglial cultures were from 1-day-old Wistar rat pups according to a previously published protocol (Fig. S1) (27). The cells were maintained in DMEM/F12 with $10 \%$ fetal bovine serum, non-essential amino acids and penicillin streptomycin (all from Gibco/Thermo Fisher Scientific) in a $\mathrm{T} 75$ flask and grown with $5 \% \mathrm{CO}_{2}$ in an incubator at $37^{\circ} \mathrm{C}$. For cell passaging, $0.05 \%$ TrypsinEDTA (Gibco/Thermo Fisher Scientific) was used for $5 \mathrm{~min}$. Microglia were visualized by immunocytochemistry for Iba-1 (rabbit polyclonal antibody, 1:500, ab108539; Abcam; goat anti-rabbit IgG (H+L) secondary antibody, Alexa Fluor 488, 1:200, A27034; Thermo Fisher Scientific). A solution of AGE in $96 \%$ ethanol was added to the culture medium to obtain a final concentration of $5 \mu \mathrm{M}$ followed by culture for $24 \mathrm{~h}$. The cells and culture medium were collected and frozen at $-70^{\circ} \mathrm{C}$ for use in enzyme-linked immunosorbent assay (ELISA).

ELISA. ELISA was carried out to quantify the hippocampal levels of interleukin (IL)-1 $\beta$, IL-10, CD86, CD163 and IL-1 $\beta$, IL-10 and CD86 levels in the microglial cell culture. The hippocampi were extracted from the left hemispheres, quickly frozen, and stored at $-70^{\circ} \mathrm{C}$ until use. Mouse IL-1 $\beta$ ELISA (ab100705), IL-10 ELISA (ab100697; both from Abcam), CD86 ELISA (KA5061) and CD163 (KA4238; both from Abnova, Taipei City, Taiwan) kits were used according to the manufacturer's recommendations. The neural tissues or cells with culture medium were homogenized on ice in the extraction buffer recommended by the manufacturer $(100 \mathrm{mM}$ Tris, pH 7.4, $150 \mathrm{mM} \mathrm{NaCl}, 1$ mM EGTA, 1 mM EDTA, $1 \%$ Triton $\mathrm{X}-100,0.5 \%$ sodium deoxycholate) with $1 \mathrm{mg} / \mathrm{ml}$ of protease inhibitor cocktail (cOmplete) and $0.01 \mathrm{mg} / \mathrm{ml}$ of phosphatase inhibitor cocktail (P5726; both from Sigma). The protein concentrations were determined using a BCA protein assay kit (Pierce, Rockford, IL, USA). The absorbance at $450 \mathrm{~nm}$ was measured with an iMark Microplate Absorbance Reader (Bio-Rad, Hercules, CA, USA).
Statistical analysis. Data are presented as the means \pm SEM. ' $n$ ' represents the number of animals for behavioral tests, and in the immunohistochemistry assay and ELISA. The data obtained by the behavioral tests, immunohistochemistry and ELISA were subjected to statistical analysis using one-way ANOVA followed by a post hoc Tukey's multiple comparison test. A value of $\mathrm{P}<0.05$ was considered to indicate a statistically significant difference. The ELISA data for cell culture were subjected to statistical analysis using a Student's t-test. All statistical tests were performed using Microsoft Excel software (Microsoft, Tulsa, OK, USA) and GraphPad prism 4 (GraphPad Software, San Diego, CA, USA).

\section{Results}

Behavioral responses to neuropathic pain and AGE treatment. To confirm the presence of neuropathic pain, cold allodynia was quantified using a 'hot/cold plate' device. The animals were placed in the apparatus, and the moment of the injured limb lifting was recorded. It was found that in the AGE-treated animals, the moment of the injured limb lifting was significantly delayed at 9 and 11 days following surgery $(\mathrm{P}<0.05)$. In the sham-operated animals, no lifting of the injured limb was observed (Fig. 1A).

The testing of locomotor activity in the 'open field' apparatus revealed a significant decrease in the number of crossed squares $(115.55 \pm 12.73$, sham-operated group vs. $80.50 \pm 11.35$, CCI group; $\mathrm{P}<0.05)$. However, in the CCI + AGE group, this indicator did not differ significantly from the level of that in the CCI group (Fig. 1B). Testing in the Y-maze revealed that sciatic nerve ligation in older animals leads to a decrease in the spontaneous alternation rate $(68.19 \pm 2.09$, sham-operated group vs. $60.12 \pm 3.36, \mathrm{CCI}$ group; $\mathrm{P}<0.05)$. In the AGE-treated animals however, a similar decrease was not observed. The behavioral indicators of the AGE-treated sham-operated animals did not differ significantly from those of the mice in the sham-operated group (Fig. 1C).

A study of long-term and short-term memory was carried out using a test termed 'novel object recognition'. The testing of short-term memory did not reveal significant differences with the sham-operated group. However, the testing of long-term memory revealed a decrease in the discrimination index in the CCI group. At the same time, an increase in time spent with the 'old' object was found. In the CCI + AGE group, no significant differences were observed with the sham-operated group. The testing of the sham-operated animals treated with AGE did not reveal any significant differences compared to the vehicle-treated sham-operated animals (Fig. 1D).

Hippocampal neurogenesis in CCI and AGE treatment. In order to determine the level of hippocampal neurogenesis in aged animals with neuropathic pain, PCNA (cell proliferation and repair marker) and DCX (a marker of newly formed neurons) were used for immunohistochemical staining. It should be noted that the expression levels of these markers in the aged animals were significantly lower than those in young animals in our previous study (19). In this study however, the induction of neuropathic pain significantly decreased the level of PCNA expression in the DG SGZ of the hippocampus $(2,268.05 \pm 427.71$, sham-operated 

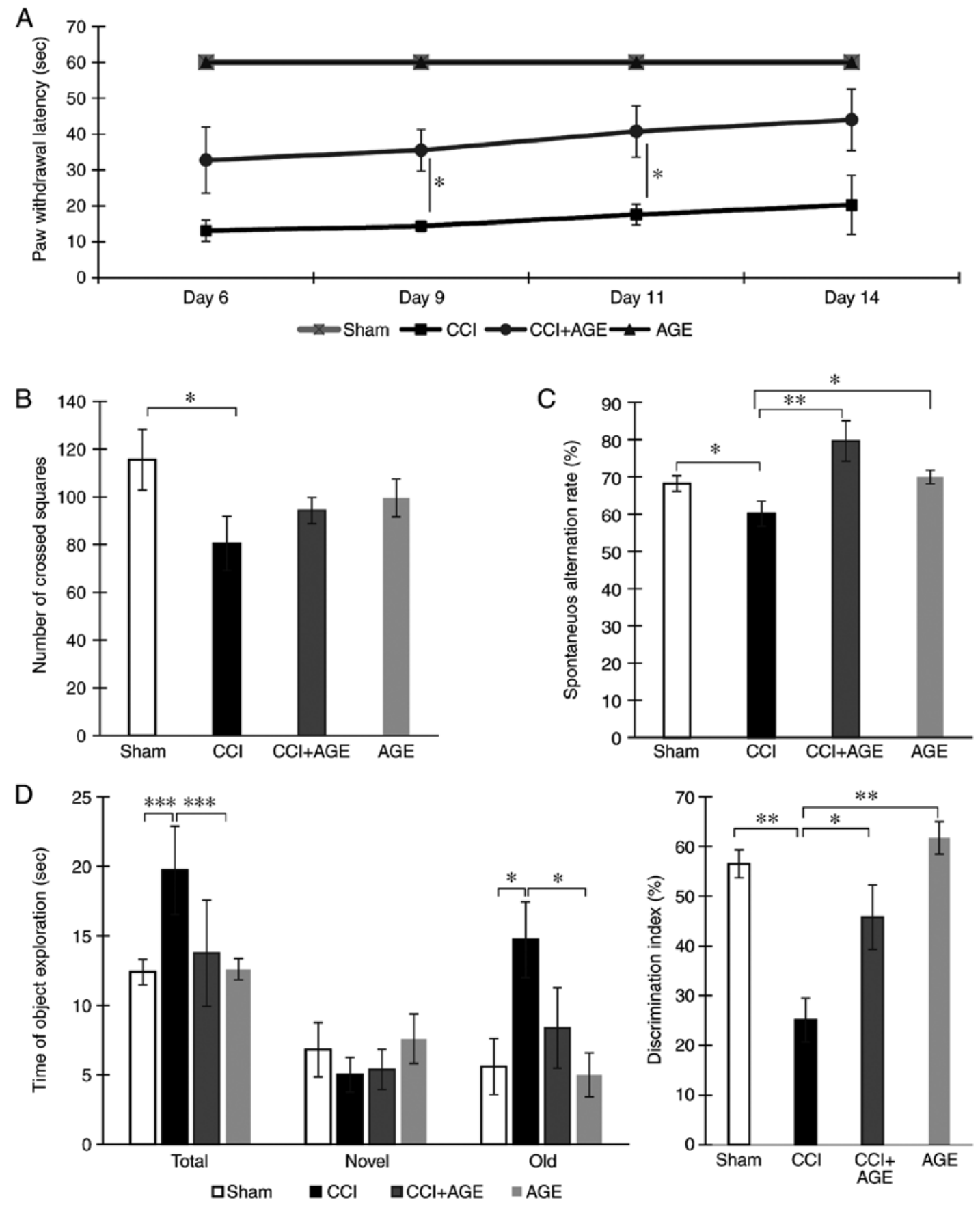

Figure 1. Behavioral effects of AGE treatment on neuropathic pain. (A) The dynamics of cold allodynia: The moment of hind paw lifting above the cold plate $\left(+4^{\circ} \mathrm{C}\right)$ for the 1-min observation. (B) Spontaneous locomotor activity in 'open field'. (C) Spatial working memory in the Y-maze. Data are the means \pm SEM, $\mathrm{n}=7$ per group, ${ }^{*} \mathrm{P}<0.05$ and ${ }^{* *} \mathrm{P}<0.01$. (D) Long-term memory: Novel object recognition with the retention period of $24 \mathrm{~h}$. Times of object exploration (left diagram); discrimination index, \% (right diagram). Data are the means $\pm \mathrm{SEM}, \mathrm{n}=7$ per group, ${ }^{*} \mathrm{P}<0.05,{ }^{* *} \mathrm{P}<0.01$ and ${ }^{* * *} \mathrm{P}<0.001$. AGE, alkyl glycerol ethers; Sham, sham-operated; CCI, chronic constriction injury.

group vs. $965.03 \pm 253.97$, CCI group; cells $/ 1 \mathrm{~mm}^{3} ; \mathrm{P}<0.05$ ). In the AGE-treated animals, no significant reduction in the number of PCNA-positive cells was observed. In addition, we found a marked decrease in DG SGZ DCX expression in the mice in the CCI group $(4,957.92 \pm 764.05$, sham-operated group vs. 2,744.27 \pm 676.06 , CCI group; cells $\left./ 1 \mathrm{~mm}^{3} ; \mathrm{P}<0.05\right)$. In the animals treated with AGE, a similar decrease was not observed $\left(2,157.27 \pm 373.49\right.$ cells $\left./ 1 \mathrm{~mm}^{3}\right)$ (Fig. 2).

Alterations in microglia changes with CCI and AGE treatment. In order to evaluate alterations in microglial activity following peripheral neurotrauma and AGE administration, we performed the immunohistochemical detection of the Iba-1-positive resident microglia pool and CD86-positive in the hippocampal areas CA1 and DG. Iba-1 is a protein that is specifically expressed in all microglia (28). CD86 is a protein expressed in pro-inflammatory microglia secreting pro-inflammatory cytokines, such as IL-6, IL-1. (29). The use of these markers allowed the quantification of any alterations in the hippocampal microglial phenotype following sciatic nerve injury and AGE treatment. We observed a significant increase in the Iba-1-positive stained area in the 

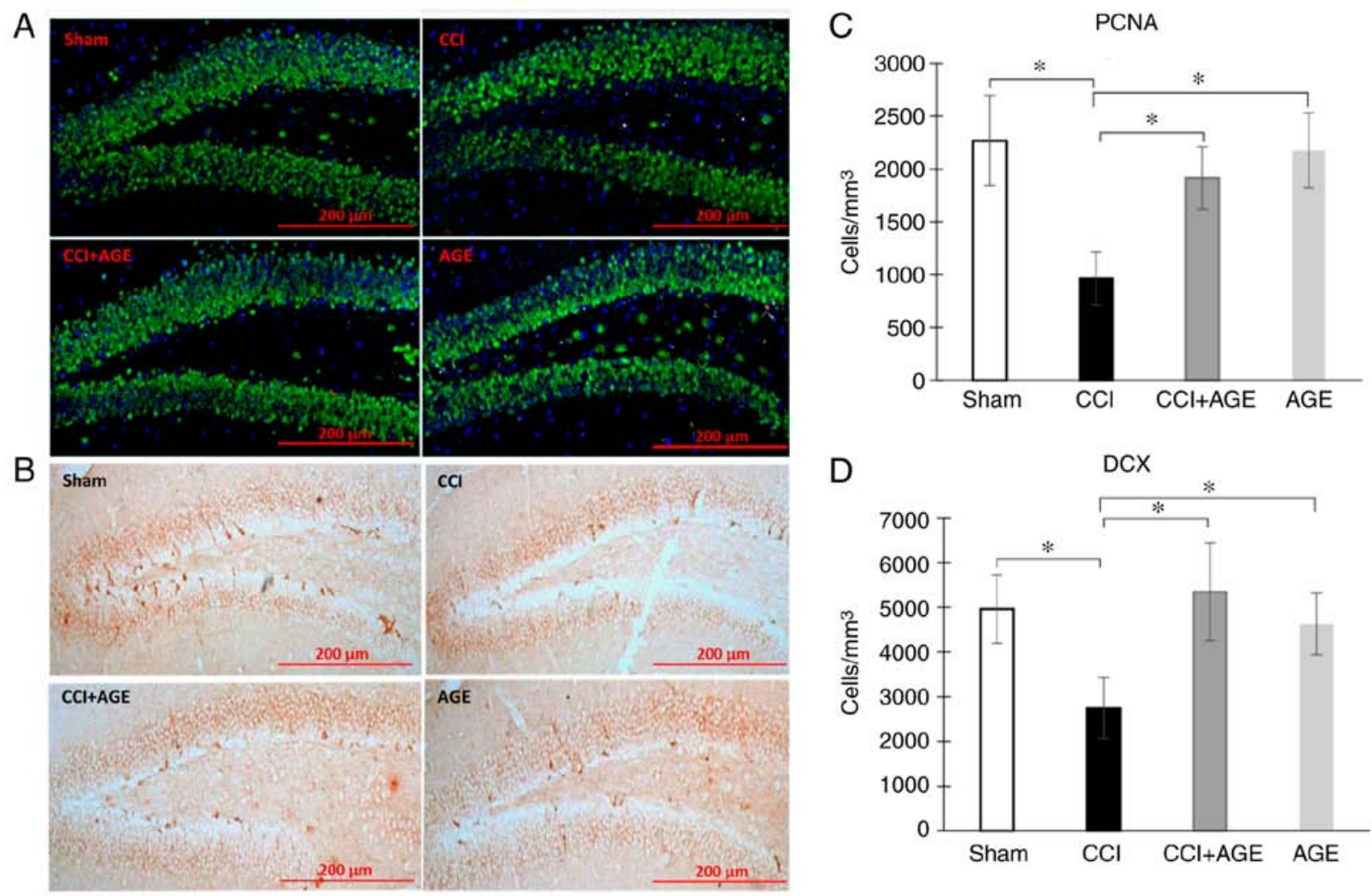

Figure 2. Proliferating cells and immature neurons in the DG SGZ. Representative images of (A) PCNA and (B) DCX expression in the SGZ of sham-operated mice, and CCI, CCI + AGE and AGE group mice. (C) Histogram showing the changes in the number of proliferating cells (by PCNA) in the DG between the sham-operated mice (red, PCNA; green, NeuN; blue, DAPI), and the CCI, CCI + AGE and AGE groups. (D) Histogram showing the changes in the number of immature neurons (by DCX) between the sham-operated mice, and the CCI, CCI + AGE and AGE group mice. Data are the means \pm SEM, $\mathrm{n}=7$ per group, ${ }^{*} \mathrm{P}<0.05$. Scale bar, $200 \mu \mathrm{m}$. Images were taken at x200 magnification. DG, dentate gyrus; SGZ, subgranular zone; PCNA, proliferating cell nuclear antigen; DCX, doublecortin; AGE, alkyl glycerol ethers; Sham, sham-operated; CCI, chronic constriction injury.

CCI group in both the CA1 region $(5.34 \pm 0.38$, sham-operated group vs. $6.73 \pm 0.51, \mathrm{CCI}$ group; $\mathrm{P}<0.05)$ and the $\mathrm{DG}$ of the hippocampus ( $4.92 \pm 0.33$, sham-operated group vs. $6.30 \pm 0.43$ CCI group; $\mathrm{P}<0.05$ ). In the animals treated with AGE following sciatic nerve lesion, an increase in Iba-1 expression was also observed $(7.83 \pm 0.71$ in the CA1; $\mathrm{P}<0.05)$. CD86 immunohistochemical detection revealed a significant increase in immunopositive staining in the CCI group compared with the sham-operated animals in the CA1 hippocampal region (13.44 \pm 0.64 , sham-operated group vs. $17.59 \pm 0.70 \mathrm{CCI}$ group; $\mathrm{P}<0.01)$ and the DG $(19.48 \pm 1.44$, sham-operated group vs. 23.44 \pm 1.36 , CCI group; $\mathrm{P}<0.05)$. In the AGE-treated animals, no significant increase in the CD86-immunopositive area staining was observed $(15.28 \pm 0.66$ in the CA1 and $19.41 \pm 1.29$ in the DG) (Fig. 3).

Hippocampal expression of inflammatory mediators and microglial/macrophage markers. Classical (M1) microglial activation is associated with the production of pro-inflammatory cytokines, such as IL-1 $\beta$, IL- 6 , tumor necrosis factor (TNF)- $\alpha$, superoxide, nitric oxide, reactive oxygen species. Alternatively (M2)-activated microglia produce anti-inflammatory cytokines, including IL-4, IL-10, transforming growth factor (TGF) $-\beta$ and insulin-like growth factor (IGF)-1 to provide tissue repair, reconstruction of the extracellular matrix and phagocytosis of cell debris (29). In this study, we used IL-1 $\beta$ and IL-6 as markers of pro-inflammatory microglia, and IL-10 as a marker of anti-inflammatory microglia. The study of hippocampal cytokine expression was carried out by ELISA. We found that the induction of neuropathic pain reduced the hippocampal levels of the pro-inflammatory cytokine, IL-1 $\beta$, at 2 weeks after surgery $(61.32 \pm 4.39$, sham-operated group vs. $48.99 \pm 2.68, \mathrm{CCI}$ group; $\mathrm{P}<0.05)$. However, in AGE-treated animals with CCI, this indicator was at the level of the sham-operated group (65.16 \pm 3.43 in CCI + AGE). Notably, a completely opposite effect was observed for another pro-inflammatory cytokine, IL-6. The induction of neuropathic pain caused an increase in the hippocampal IL-6 level (5.86 \pm 0.95 , sham-operated group vs. 13.27 \pm 2.43 , CCI group; $\mathrm{P}<0.05)$, while AGE administration prevented the development of this effect (6.17 $\pm 2.10, \mathrm{CCI}+$ AGE group). The study of the expression of the anti-inflammatory cytokine, IL-10, revealed a decrease in mice with neuropathic pain $(56.25 \pm 16.875$, sham-operated group vs. $17.37 \pm 4.73, \mathrm{CCI}$ group), and in the AGE-treated animals, the expression was at the level of that in the sham-operated group (Fig. 4A).

The study of hippocampal microglial/macrophage markers using ELISA revealed an increased expression of CD86 $(65.06 \pm 2.48, \mathrm{CCI}$ group vs. 76.76 $\pm 2.34, \mathrm{CCI}+\mathrm{AGE}$ group; $\mathrm{P}<0.01$ ), whereas in the AGE-treated mice, an increase in this protein was not observed. The study of hippocampal CD163 expression, a marker of anti-inflammatory microglial/macrophages, revealed a significant increase in the animals with CCI $(\mathrm{P}<0.01)$. At the same time, in the AGE-treated mice with 

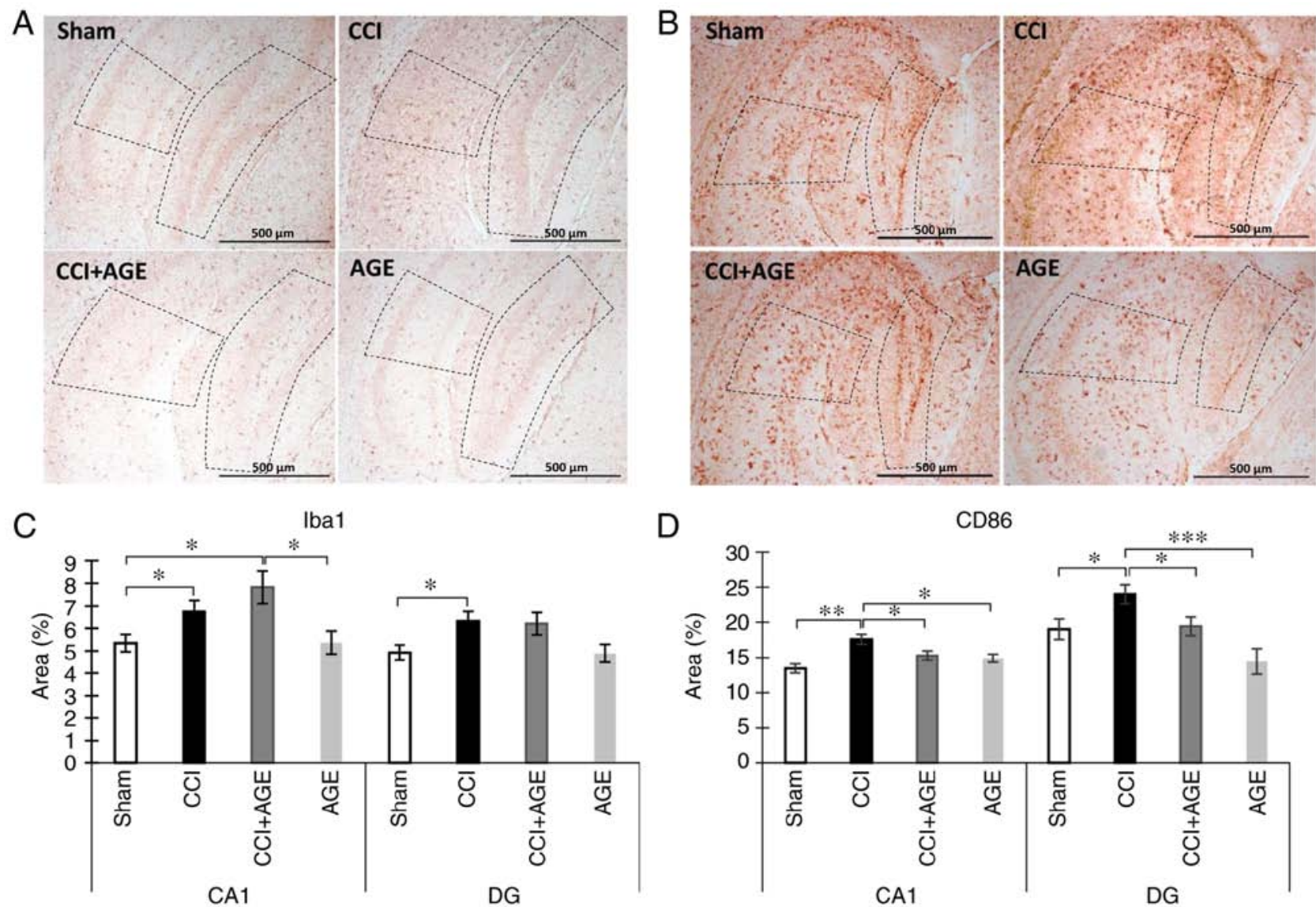

$\mathrm{D}$

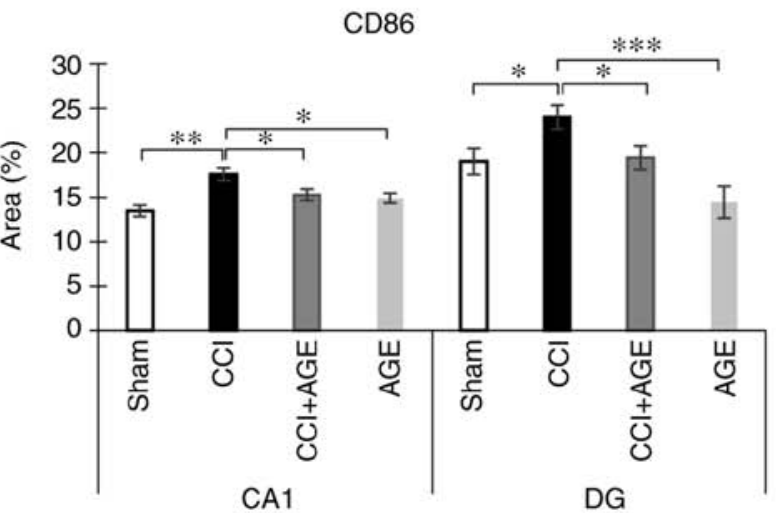

Figure 3. Effects of CCI and AGE treatment on microglial activation. (A) Representative images of Iba-1 immunohistochemically-stained slides. (B) Representative images of CD86 immunohistochemically-stained slides. (C) Histogram demonstrating the percentage of area covered by Iba-1-positive staining in the CA1 hippocampal region at 14 days after surgery. Data are the means \pm SEM, $n=7$ per group, ${ }^{*} \mathrm{P}<0.05$. (D) Histogram demonstrating the percentage of area covered by CD86-positive staining in the CA1 hippocampal region at 14 days after surgery, $\%$. Data are the means \pm SEM, $n=7$ per group ${ }^{*} \mathrm{P}<0.05,{ }^{* *} \mathrm{P}<0.01$ and ${ }^{* * *} \mathrm{P}<0.001$. Scale bar, $500 \mu \mathrm{m}$. Images were taken at $\mathrm{x} 100$ magnification. The dotted line indicates the boundaries of the CA1 and DG regions. AGE, alkyl glycerol ethers; Sham, sham-operated; CCI, chronic constriction injury; DG, dentate gyrus.

CCI, CD163 was also significantly higher than the levels in the sham-operated group $(\mathrm{P}<0.01)$ (Fig. 4B).

Effects of AGE treatment on microglial cytokine production in vitro. To determine the effects of AGE treatment on the state of microglial cells in vitro, the drug was added to the culture medium at a concentration of $5 \mu \mathrm{M}$ and after $24 \mathrm{~h}$, the culture medium with the cells was examined by ELISA. It was found that AGE treatment increased the production of the pro-inflammatory cytokine, IL-1 $\beta$, by $40 \%$. However, the concentration of IL-10 remained unaltered. In addition, a slight, yet significant increase in the expression of the pro-inflammatory microglial marker, CD86, was found $(\mathrm{P}<0.05)$. Thus, AGE treatment induced microglial M1-like polarization in our in vitro experiment (Fig. 5).

\section{Discussion}

In this study, we examined the effects of neuropathic pain and AGE therapy on the state of microglia, neurogenesis in the hippocampus and pain behavior in aged mice. The use of 18-month-old animals is beneficial due to reduced neurogenesis intensity (30). This circumstance, as expected, allows us to obtain a clearer picture of immunohistochemical and biochemical changes observed following sciatic nerve ligation. Of note, in some cases, the age of the animals contributes to the development of opposite effects compared to our previous results obtained in young animals (19). Namely, in 18-month-old mice, the induction of neuropathic pain caused a decrease in locomotor activity, while in three-month-old mice an increase in locomotor activity was observed. Such age-related features are usually associated with altered neurotransmission, as well as the sensitivity of receptors involved in pain perception and modulation. In addition, a similar effect may be associated with age-related changes in the number of excitatory and inhibitory synapses on neurons (12). These factors affect the manifestation of pain-related behaviors exhibited by experimental animals, depending on age. However, in both cases, significant changes in the spatial working memory rate are observed, which emphasizes the involvement of the hippocampal formation in the pathogenesis of neuropathic pain. The violation of spatial orientation due to the involvement of the hippocampus can be explained by the presence of direct axonal projections from layer III of the medial entorhinal cortex into CA1 hippocampal region.

In addition to working spatial memory, in this study, we investigated long-term memory in mice with CCI and AGE treatment. We observed a decrease in the discrimination index in animals with CCI. Moreover, the index was reduced by 
A

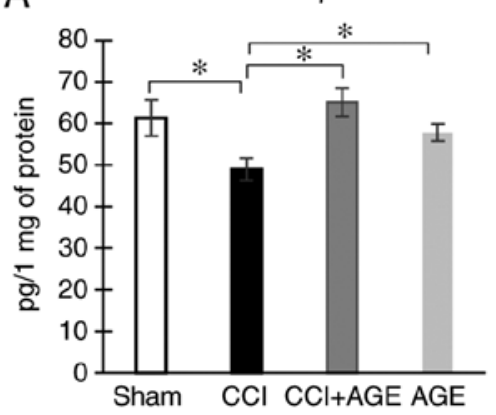

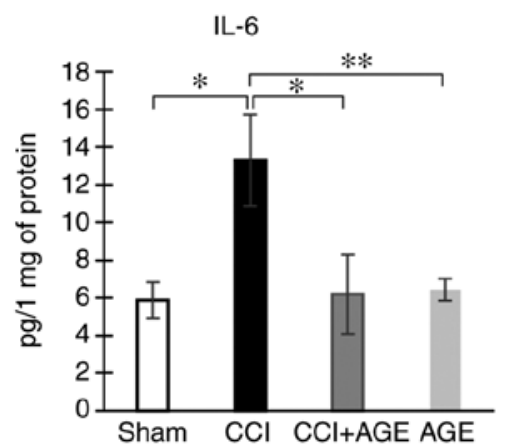

IL-10

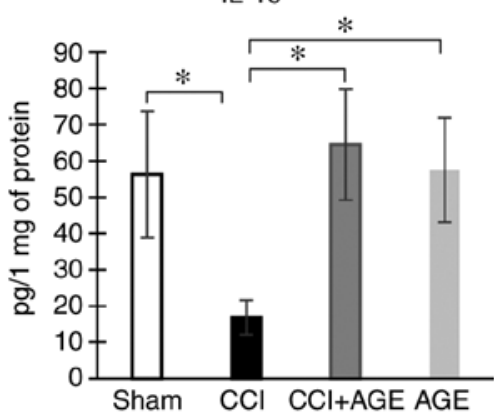

$\mathrm{B}$

CD86

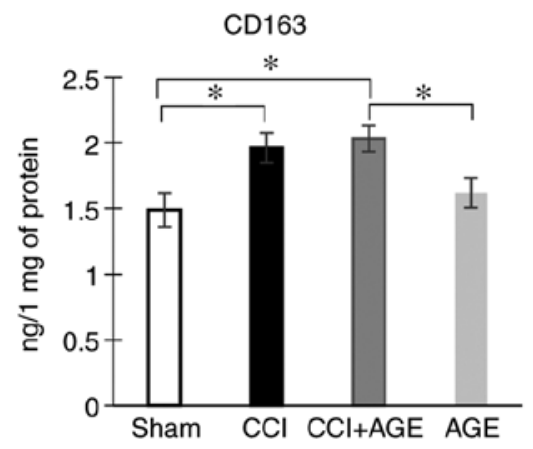

Figure 4. Effects of CCI and AGE treatment on hippocampal cytokine and microglial marker expression. (A) Histograms of Il-1 $\beta$, Il-6 and Il-10 hippocampal expression. Data are the means $\pm \mathrm{SEM}, \mathrm{n}=7$ per group, ${ }^{*} \mathrm{P}<0.05$ and ${ }^{* *} \mathrm{P}<0.01$. (B) Histograms of CD86 and CD163 hippocampal expression. Data are the means \pm SEM, $n=7$ per group, ${ }^{*} \mathrm{P}<0.05$ and ${ }^{* *} \mathrm{P}<0.01$. AGE, alkyl glycerol ethers; Sham, sham-operated; CCI, chronic constriction injury; IL, interleukin.

$\mathrm{IL}-1 \beta$

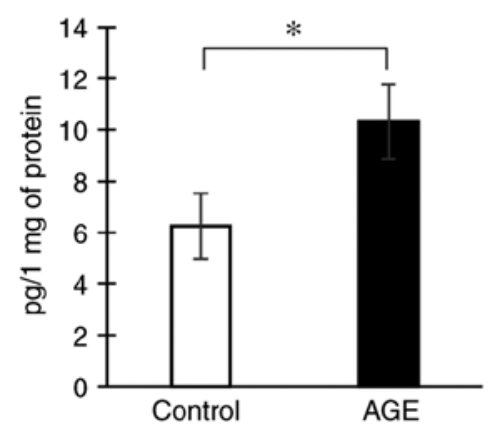

IL-10

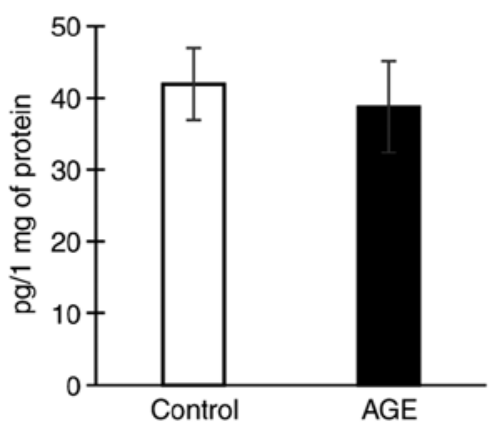

CD86

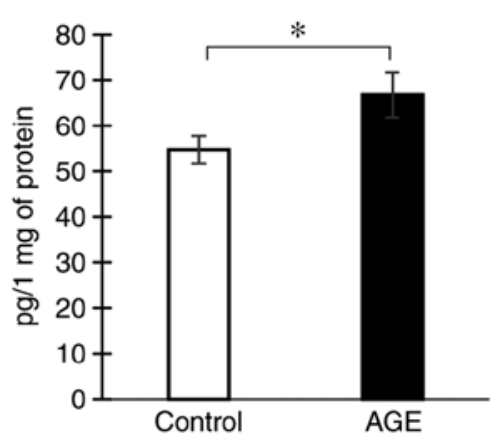

Figure 5. IL-1 $\beta$, IL-10 and CD86 expression in microglial cell culture, $\mathrm{n}=5$ per group (number of tested wells with cells), ${ }^{*} \mathrm{P}<0.05$. IL, interleukin; AGE, alkyl glycerol ethers.

increasing the time spent with the 'old' object in animals with CCI. We hypothesized that this may occur due to a decrease in locomotor activity in animals with neuropathic pain and as a result increase in time spent with objects. Thus, the result certainly suggests that neuropathic pain leads to long-term memory impairment, and AGE prevents this effect. However, we do not exclude the effect of reduced locomotor activity on this indicator.

The observed changes in glial marker expression may be a consequence of those anatomical connections associated with pain ascending systems (4). Thus, an increase in the total microglia population (Iba- $1^{+}$cells) percentage with a simultaneous increase in the activation of proinflammatory M1 microglia was found 2 weeks after surgery in CA1 and DG hippocampal regions. According to the testing on the hot plate, this time was accompanied by a maximum pain response to the nerve ligation among the entire observation period.
AGE treatment prevented the activation of pro-inflammatory (M1) microglia with a simultaneous increase in the number of Iba1-positive microglia. This may indirectly indicate the phenotypic conversion of microglia to the anti-inflammatory phenotype. The results of the ELISA confirm this assumption, demonstrating that AGE decreased the levels of the pro-inflammatory cytokine, IL-6, and increased those of the anti-inflammatory cytokine, IL-10, within the hippocampus. The results of immunohistochemistry were also confirmed by ELISA, which demonstrated an increase in the CD86 concentration in the CCI group and the prevention of such an increase in the AGE-treated mice. However, an interesting result was obtained as regards the pro-inflammatory cytokine, IL-1 $\beta$. The induction of neuropathic pain caused a significant decrease in its production, while in the AGE-treated animals, no significant decrease was observed. A similar result has also been obtained previously (31), when a trend for decreased 
IL-1 $\beta$ was observed within the thalamus at 10 days after surgery. However, the mechanisms of this phenomenon remain unclear. Nevertheless, it is known that the presence of IL-1 $\beta$ is necessary to maintain a normal long-term potentiation, which emphasizes the involvement of this cytokine in the processes of memory and learning (32). Thus, in young animals, the increase in IL-1 $\beta$ levels in response to painful stimuli is adaptive, while in aged animals, the production of this cytokine is impaired due to impaired adaptation processes.

We also obtained an interesting result regarding the anti-inflammatory cytokine, IL-10. In older mice, a decrease in the concentration of IL-10 was found in the present study, while in young animals with neuropathic pain an increase was previously observed (19). This may also indicate a disruption of the adaptive mechanisms in older animals. In addition, an age-related decrease in IL-10 production in the mouse brain has been shown to trigger a cascade of intracellular events, leading to an increase in IL-6 gene expression (33). However, in this study, in animals treated with AGE, the concentration of IL-10 in the hippocampus remained at the level of the sham-operated group. The ELISA data demonstrating the concentration of the anti-inflammatory microglia/macrophage marker CD163 for pain and AGE administration also give rise to speculation. Despite a decrease in IL-10 levels in animals with neuropathic pain, we observed an increase in the CD163 levels. At the same time, AGE administration had no effect on the concentration of this marker. Perhaps this result is a consequence of the fact that CD163-positive cells are perivascular macrophages (34) and reflect the peripheral immune response to the AGE administration. In addition, it is possible that an increase in the production of IL- $1 \beta$ occurs precisely due to the migration of peripheral macrophages. This can be facilitated by AGE administration, which is known to be able to increase the blood-brain barrier permeability [reviewed in $(35)]$.

To confirm the hypothesis about the induction of M2 microglial activation under the influence of AGE treatment, we performed in vitro experiments using microglial cell culture. Surprisingly, the addition of AGE to the culture medium at a concentration of $5 \mu \mathrm{M}$ resulted in an increase in IL-1 $1 \beta$ production by microglial cells. In this case, the concentration of IL-10 in cells and culture medium remained unchanged upon addition of AGE. At the same time, we found an increase in CD86 concentration, which was shown by ELISA. These data are consistent with those found in the literature on the effect of AGE on cytokine production by macrophages in vitro. For example, AGE contained in shark liver oil has been shown to lead to an increase in interferon (IFN) $-\gamma$ production, without affecting the IL-4 level in splenic mononuclear cells (36). However, our in vivo immunohistochemical study revealed no significant changes in the expression of glial markers, as well as pro-inflammatory cytokines in AGE-treated sham-operated group (AGE) compared to the sham-operated group. This indicates that in vivo, the influence of AGE occurs indirectly, which makes it possible to regulate already impaired functions and not to affect the normally functioning organism.

The observed changes in the microglial state were accompanied by a decrease in the intensity of hippocampal neurogenesis during pain. At the same time, the basic level of neurogenesis in aged mice was significantly lower than that in young animals in our previous experiments (19). However, the administration of AGE to animals prevented a decrease in both the number of proliferating cells and the number of newly formed neurons. Probably, this phenomenon is associated with a change in the state of microglia, its pro- or anti-inflammatory transformation and the spectrum of produced cytokines. As it is known, pro-inflammatory cytokines inhibit the processes of neurogenesis. For example, in transgenic mice with an increased production of IL- 6 by astrocytes, a decrease in the proliferative activity in the SGZ neurons has been observed (37). At the same time, an increase in the production of anti-inflammatory cytokines is usually associated with the stimulation of neurogenesis $(38,39)$. The observed effects of neuropathic pain and treatment with AGE on working and long-term memory may be the result of changes in neurogenesis (40).

The data on the pharmacological effects of AGE, in particular the effect on the polarization of microglia, the spectrum of produced cytokines and hippocampal neurogenesis indicate the neuroprotective properties of this drug. However, the opposite data obtained in in vivo and in vitro experiments suggest the idea that there is no direct effect of AGE on the brain microglial cells within a living organism. We still aim to study the indirect mechanisms of AGE influence on the microglial state and the processes of neurogenesis, although we assume several possible mechanisms. For example, it was previously shown that the administration of AGE to animals leads to an increase in the plasmalogen concentration in various tissues (41). It is the pronounced anti-inflammatory activity of plasmalogen (42) that can underlie the observed decrease in the pro-inflammatory activation of microglia and the maintaining of the normal neurogenesis level. In general, we assume that the effect of AGE on the central nervous system is complex and is implemented through several mechanisms, including regulation of plasmalogen synthesis, neurotrophic factors production, the blood-brain barrier permeability, and several other mechanisms that require detailed study.

In conclusion, neuropathic pain resulting from disturbances in the peripheral nervous system often causes the development of pathological processes of the central nervous system. This study emphasizes that pain-induced behavioral deficit is based on a neuroinflammatory response that covers primarily the limbic system including the hippocampus, which is involved in neuroplasticity, behavior, and cognition. Thus, an effective treatment for the neuropathic pain cognitive and emotional consequences may be precisely the effect on the functional activity of brain resident macrophages. The drug based on AGE that we study can affect the functions of microglial cells both in vitro and in vivo. Taken together, these data suggest that AGE prevent neuropathic pain-derived effects, including M1 microglial activation, neurogenesis disruption and memory disturbances. At the same time, the results of the in vitro experiments emphasize the complexity of the mechanisms underlying AGE pharmacological effects. This fact indicates AGE therapeutic potential and the promise of its further study in terms of the neuropathic pain pharmacological management.

\section{Acknowledgements}

Not applicable. 


\section{Funding}

The present study was supported by the Russian Science Foundation (project no. 17-74-20006).

\section{Availability of data and materials}

All the data generated and analyzed in the present study are available from the corresponding author upon reasonable request.

\section{Authors' contributions}

AT and IM designed the study. AT and IM performed the surgery. EE prepared the alkyl glycerol ethers. IM performed the immunohistochemical analysis. AT performed the behavioral tests, ELISA and image analysis. YK performed the in vitro experiments. AT wrote the manuscript. All authors have read and approved the final manuscript.

\section{Ethics approval and consent to participate}

All procedures were approved by the Animal Ethics Committee at National Scientific Center of Marine Biology Far Eastern Branch, Russian Academy of Sciences, according to the Laboratory Animal Welfare guidelines.

\section{Patient consent for publication}

Not applicable.

\section{Competing interests}

The authors declare that they have no competing interests to disclose.

\section{References}

1. Merskey H and Bogduk N: Pain terms: A current list with definitions and notes on usage: Classification of chronic pain, 2nd ed; Seattle: IASP Task Force on Taxonomy, USA, pp. 154-196, 1994 ISBN.

2. Baron R, Binder A and Wasner G: Neuropathic pain: Diagnosis, pathophysiological mechanisms, and treatment. Lancet Neurol 9: 807-819, 2010

3. McEwen BS: Plasticity of the hippocampus: Adaptation to chronic stress and allostatic load. Ann N Y Acad Sci 933: 265-277, 2001.

4. Liu MG and Chen J: Roles of the hippocampal formation in pain information processing. Neurosci Bull 25: 237-266, 2009.

5. Deng W, Aimone JB and Gage FH: New neurons and new memories: How does adult hippocampal neurogenesis affect learning and memory? Nat Rev Neurosci 11: 339-350, 2010.

6. Sahay A and Hen R: Adult hippocampal neurogenesis in depression. Nat Neurosci 10: 1110-1115, 2007.

7. Revest JM, Dupret D, Koehl M, Funk-Reiter C, Grosjean N, Piazza PV and Abrous DN: Adult hippocampal neurogenesis is involved in anxiety-related behaviors. Mol Psychiatry 14: 959-567, 2009

8. Dranovsky A and Hen R: Hippocampal neurogenesis: Regulation by stress and antidepressants. Biol Psychiatry 59: 1136-1143, 2006.

9. Saxe MD, Battaglia F, Wang JW, Malleret G, David DJ, Monckton JE, Garcia AD, Sofroniew MV, Kandel ER, Santarelli L, et al: Ablation of hippocampal neurogenesis impairs contextual fear conditioning and synaptic plasticity in the dentate gyrus. Proc Natl Acad Sci USA 103: 17501-17506, 2006.
10. Argoff CE: The coexistence of neuropathic pain, sleep, and psychiatric disorders: A novel treatment approach. Clin J Pain 23: 15-22, 2007.

11. Fillingim RB, King CD, Ribeiro-Dasilva MC, Rahim-Williams B and Riley JL III: Sex, gender, and pain: A review of recent clinical and experimental findings. J Pain 10: 447-485, 2009.

12. Leite-Almeida H, Almeida-Torres L, Mesquita AR, Pertovaara A, Sousa N, Cerqueira JJ and Almeida A: The impact of age on emotional and cognitive behaviours triggered by experimental neuropathy in rats. Pain 144: 57-65, 2009.

13. Reicherts P, Gerdes AB, Pauli P and Wieser MJ: Psychological placebo and nocebo effects on pain rely on expectation and previous experience. J Pain 17: 203-214, 2016.

14. Li Q, Navakkode S, Rothkegel M, Soong TW, Sajikumar S and Korte M: Metaplasticity mechanisms restore plasticity and associativity in an animal model of Alzheimer's disease. Proc Natl Acad Sci USA 114: 5527-5532, 2017.

15. Lister JP and Barnes CA: Neurobiological changes in the hippocampus during normative aging. Arch Neurol 66: 829-833, 2009.

16. Schmidt M, Dubin AE, Petrus MJ, Earley TJ and Patapoutian A: Nociceptive signals induce trafficking of TRPA1 to the plasma membrane. Neuron 64: 498-509, 2009.

17. McQuail JA, Frazier CJ and Bizon JL: Molecular aspects of age-related cognitive decline: The role of GABA signaling. Trends Mol Med 21: 450-460, 2015.

18. Jones MR, Ehrhardt KP, Ripoll JG, Sharma B, Padnos IW, Kaye RJ and Kaye AD: Pain in the elderly. Curr Pain Headache Rep 20: 23, 2016

19. Tyrtyshnaia AA, Manzhulo IV, Sultanov RM and Ermolenko EV: Adult hippocampal neurogenesis in neuropathic pain and alkyl glycerol ethers treatment. Acta Histochem 119: 812-821, 2017.

20. Bennett GJ and Xie YK: A peripheral mononeuropathy in rat that produces disorders of pain sensation like those seen in man. Pain 33: 87-107, 1988.

21. Bligh EG and Dyer WJ: A rapid method of total lipid extraction and purification. Can J Biochem Physiol 37: 911-917, 1959.

22. Ermolenko EV, Latyshev NA, Sultanov RM and Kasyanov SP: Technological approach of 1-O-alkyl-sn-glycerols separation from Berryteuthis magister squid liver oil. J Food Sci Technol 53: 1722-1726, 2016.

23. Allen JW and Yaksh TL: Assessment of acute thermal nociception in laboratory animals. Methods Mol Med 99: 11-23, 2004.

24. Manzhulo IV, Ogurtsova OS, Kipryushina YO, Latyshev NA, Kasyanov SP, Dyuizen IV and Tyrtyshnaia AA: Neuron-astrocyte interactions in spinal cord dorsal horn in neuropathic pain development and docosahexaenoic acid therapy. J Neuroimmunol 298: 90-97, 2016.

25. Lysenko LV, Kim J, Henry C, Tyrtyshnaia A, Kohnz RA, Madamba F, Simon GM, Kleschevnikova NE, Nomura DK, Ezekowitz RA and Kleschevnikov AM: Monoacylglycerol lipase inhibitor JZL184 improves behavior and neural properties in Ts65Dn mice, a model of down syndrome. PLoS One 9: e114521, 2014.

26. Bevins RA and Besheer J: Object recognition in rats and mice: A one-trial non-matching-to-sample learning task to study 'recognition memory'. Nat Protoc 1: 1306-1311, 2006.

27. Saura J, Tusell JM and Serratosa J: High-yield isolation of murine microglia by mild trypsinization. Glia 44: 183-189, 2003.

28. Ahmed Z, Shaw G, Sharma VP, Yang C, McGowan E and Dickson DW: Actin-binding proteins coronin-1a and IBA-1 are effective microglial markers for immunohistochemistry. J Histochem Cytochem 55: 687-700, 2007.

29. Tang YU and Weidong LE: Differential roles of M1 and M2 microglia in neurodegenerative diseases. Mol. Neurobiol 53: 1181-1194, 2016.

30. Van Praag H, Shubert T, Zhao C and Gage FH: Exercise enhances learning and hippocampal neurogenesis in aged mice. J Neurosc 25: 8680-8685, 2005.

31. Apkarian AV, Baliki MN and Geha PY: Towards a theory of chronic pain. Prog Neurobiol 87: 81-97, 2009.

32. Schneider H, Pitossi F, Balschun D, Wagner A, Del Rey A and Besedovsky HO: A neuromodulatory role of interleukin-1 $\beta$ in the hippocampus. Proc Natl Acad Sci USA 95: 7778-7783, 1998.

33. YeSMand Johnson RW: An age-related decline in interleukin-10 may contribute to the increased expression of interleukin-6 in brain of aged mice. Neuroimmunomodulation 9: 183-192, 2001. 
34. Galea J, Cruickshank G, Teeling JL, Boche D, Garland P, Perry VH and Galea I: The intrathecal CD163-haptoglobin-hemoglobin scavenging system in subarachnoid hemorrhage. J Neurochem 121: 785-792, 2012

35. Iannitti T and Palmieri B: An update on the therapeutic role of alkylglycerols. Mar Drugs 8: 2267-2300, 2010.

36. Hajimoradi M, Hassan ZM, Pourfathollah AA, Daneshmandi S and Pakravan $\mathrm{N}$ : The effect of shark liver oil on the tumor infiltrating lymphocytes and cytokine pattern in mice. J Ethnopharmacol 126: 565-570, 2009.

37. Vallieres L, Campbell IL, Gage FH and Sawchenko PE: Reduced hippocampal neurogenesis in adult transgenic mice with chronic astrocytic production of interleukin-6. J Neurosci 22: 486-492, 2002.

38. Perez-Asensio FJ, Perpiñá U, Planas AM and Pozas E: Interleukin-10 regulates progenitor differentiation and modulates neurogenesis on adult brain. J Cell Sci 126: 4208-4219, 2013.

39. Pereira L, Font-Nieves M, Van den Haute C, Baekelandt V, Planas AM and Pozas E: IL-10 regulates adult neurogenesis by modulating ERK and STAT3 activity. Front Cell Neurosci 25 : 9-57, 2015 .
40. McKim DB, Niraula A, Tarr AJ, Wohleb ES, Sheridan JF and Godbout JP: Neuroinflammatory dynamics underlie memory impairments after repeated social defeat. J Neurosci 36: 2590-2604, 2016.

41. Brites P, Ferreira AS, Da Silva TF, Sousa VF, Malheiro AR, Duran M, Waterham HR, Baes M and Wanders RJ: Alkyl-glycerol rescues plasmalogen levels and pathology of ether-phospholipid deficient mice. PLoS One 6: e28539, 2011.

42. Ifuku M,KatafuchiT, Mawatari S, Noda M, Miake K, Sugiyama M and Fujino T: Anti-inflammatory/anti-amyloidogenic effects of plasmalogens in lipopolysaccharide-induced neuroinflammation in adult mice. J Neuroinflammation 9: 197, 2012.

This work is licensed under a Creative Commons Attribution-NonCommercial-NoDerivatives 4.0 International (CC BY-NC-ND 4.0) License. 